Thorax (1973), 28, 613.

\title{
A new surgical approach to myocardial revascular- ization-internal mammary artery to coronary vein anastomosis ${ }^{1}$
}

\author{
ISMAIL A. SALLAM ${ }^{2}$ and WILLEM J.KOLFF \\ Division of Artificial Organs, Department of Surgery and Institute of Biomedical Engineering, \\ University of Utah, Salt Lake City, Utah, U.S.A.
}

\begin{abstract}
Sallam, I. A., and Kolff, W. J. (1973). Thorax, 28, 613-616. A new surgical approach to myocardial revascularization-internal mammary artery to coronary vein anastomosis. Myocardial ischaemia was established in anaesthetized animals (five dogs and five sheep) by ligating the anterior descending interventricular branch of the left coronary artery. Once the ischaemic changes became evident electrocardiographically, retrograde perfusion of the coronary vein draining the ischaemic area was established by connecting the internal mammary artery to the coronary vein. The vein was ligated proximally.

When retrograde perfusion of the coronary vein was begun, the signs of ischaemia gradually disappeared but could be reproduced by clamping the internal mammary artery. This indicated the effectiveness of the anastomosis in establishing an efficient new blood supply to the ischaemic area.

The present approach offers a new prospect to patients in whom the coronary artery is not suitable for coronary vein bypass grafting due to extensive diffuse narrowing. Further application may be found in patients with cardiogenic shock after myocardial infarction. Direct immediate revascularization by this approach may prove of value in such cases.
\end{abstract}

Coronary bypass grafting has been almost established as an effective and safe measure in treating selected cases of myocardial ischaemia (Favaloro, 1969 ; Johnson and Lepley, 1970 ; Spencer, 1970 ; Mitchel et al., 1970). For success of the bypass graft operations accurate selection of cases is a prerequisite. When the coronary artery is badly diseased and the lumen is obliterated, the operation has been unsuccessful in a high proportion of cases (Mitchel et al., 1970 ; Spencer, 1970 ; Adam, Mitchel, Lambert, and Geisler, 1972). The possibility of augmentation of the coronary blood flow in such cases by utilizing the coronary vein instead of the artery is considered in this study. Our results show that perfusion of the coronary vein by the internal mammary artery proves to be of significant value in ameliorating the effects of acute myocardial ischaemia in animals after ligating the

1This work was supported by Grant No. HL-13738-OIAI of the National Heart and Lung Institute of the National Institute of Health, U.S.A.

2Reprint requests : Mr. I. A. Sallam, Kuwait Chest Hospital, Kuwait POB5 anterior descending coronary artery. This may also suggest the feasibility of employing such procedures in treating some patients presenting with cardiogenic shock after myocardial infarction.

\section{MATERIALS AND METHODS}

Studies were made on 10 animals, five sheep weighing $62-72 \mathrm{~kg}$ and five mongrel dogs weighing $25-28 \mathrm{~kg}$. Induction of anaesthesia was by intravenous sodium methohexital ( $5 \mathrm{mg} / \mathrm{kg}$ of body weight) and scopolamine $(0.03 \mathrm{mg} / \mathrm{kg}$ of body weight). An endotracheal tube was inserted and ventilation was controlled with a Bird respirator. Anaesthesia was maintained with nitrous oxide, oxygen, and a methoxyflurane mixture.

The left femoral vessels were cannulated. The femoral artery was used to monitor the systemic arterial blood pressure. The central venous pressure was measured by a cannula reaching the inferior vena cava via the femoral vein. The electrocardiogram, central venous pressure, arterial blood pressure, and left atrial pressure were continuously monitored.

In dogs, the chest was opened through a left anterolateral thoracotomy in the fourth intercostal space; a sternal split incision was used in sheep. An epicardial 
electrocardiographic lead was attached near the apex. The anterior descending interventricular coronary artery was dissected. A ligature was applied $3.5 \mathrm{~cm}$ below its origin from the left coronary artery. When ischaemic manifestations became evident from electrocardiographic and/or haemodynamic changes, an attempt to perfuse the coronary vein was started. Perfusion of the coronary vein was accomplished either by direct end-to-side anastomosis (in two cases) or by the interposition of a Teflon cannula between the internal mammary artery and the coronary vein after ligating the proximal end of the vein. The blood flow through the anastomosis could be controlled by a clamp applied across the internal mammary artery (Fig. 1).

When perfusion of the coronary vein was begun, the signs of ischaemia disappeared over a period of 15-25 minutes but could be reproduced by clamping the internal mammary artery. Continuous monitoring was maintained throughout the procedure.

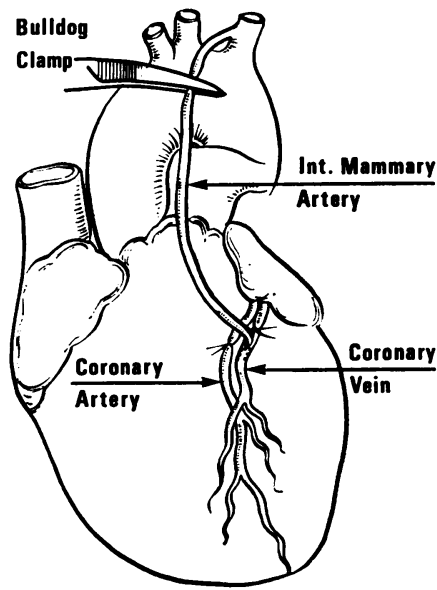

FIG. 1. Diagram showing the completed procedure.
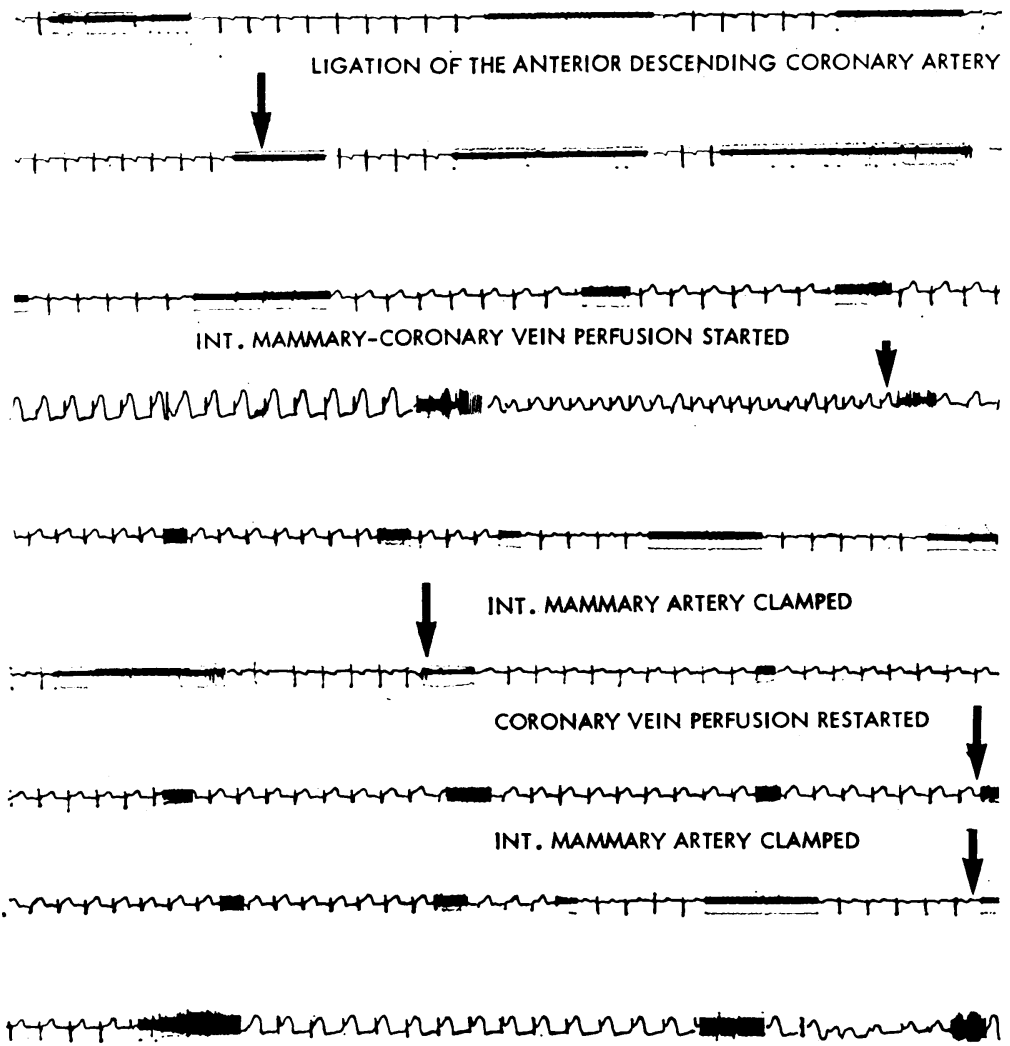

FIG. 2. ECG epicardial lead. Effects of coronary vein perfusion on myocardial ischaemia induced by ligation of the anterior descending coronary artery. 


\section{RESULTS}

Ligation of the anterior descending coronary artery resulted in ischaemic changes. Two animals died from ventricular fibrillation consequent on the coronary artery ligation. In the rest, anterolateral ischaemic changes were evident, especially in lead I, AVL, and the epicardial lead, in the form of elevation of the ST segment with inversion of the $T$ wave. Perfusion of the coronary vein from the internal mammary artery was followed by gradual improvement in the ischaemic changes (Fig. 2). If, at this point, the internal mammary was clamped, ischaemic changes again developed. The process was reversible in all the animals used and could be repeated from three to six times.

Haemodynamic changes were variable, apart from changes in the arterial blood pressure which were consistent. Induction of myocardial ischaemia produced a variable drop in the blood pressure shortly after the ischaemic changes were evident in the electrocardiogram. The blood pressure returned to its original level gradually with coronary vein perfusion. The left atrial pressure was raised in three out of eight animals after ligation of the artery. Establishment of the anastomosis resulted in lowering of the left atrial pressure accompanied by the disappearance of the electrocardiographic changes in all the cases in which the left atrial pressure was raised.

\section{DISCUSSION}

Implantation of the internal mammary artery into the coronary vein in these experiments appeared to be effective in increasing the blood supply to the ischaemic area produced by ligation of the anterior descending coronary artery as evidenced by regression of the electrocardiographic changes. Haemodynamic changes, on the other hand, were not significant in all the cases but they were reversible whenever they occurred. The reproduction of ischaemic changes following clamping of the internal mammary artery indicated the effectiveness of the anastomosis in establishing an efficient new blood supply to the ischaemic area and seemed to exclude the possibility that recovery obtained was merely a part of the natural recovery process.

Internal mammary artery/coronary vein end-toside anastomosis is technically difficult in dogs and sheep, in which the vein wall is thin; however, previous experience at the same centre on calves had indicated the feasibility of the pro- cedure. Previous attempts to utilize the coronary sinus were described by Beck et al. in 1948 ; they placed a vein graft between the aorta and the coronary sinus as a first stage, followed a month later by a second stage in which the coronary sinus is narrowed (Beck, Stanton, Batiuchok, and Leiter, 1948). The high mortality rate made such a procedure unacceptable (Favaloro, 1970). The present approach offers a relatively simple procedure, which may provide new prospects in two groups of patients, in whom neither available surgical nor medical treatment proves to be of value. The first group includes patients in whom the coronary artery is not suitable for coronary bypass vein grafts due to extensive diffuse narrowing. The vein in such patients can provide an alternative channel; even a more proximal anastomosis will be possible, providing an increase in the blood supply to a larger area of the myocardium.

The other group includes those patients presenting with cardiogenic shock after acute myocardial infarction. Results of medical treatment in these patients are disappointing, as the average mortality rate is about $80 \%$ (Kuhn, 1970). Although the shock syndrome may present different haemodynamic patterns, as shown in this study or in previous reports (Nixon, 1968; Nixon, Taylor, and Morton, 1968 ; Begg et al., 1969 ; Lassers et al., 1970), in all cases it reflects the disproportion of the oxygen need of the infarcted area and the available blood supply (Kuhn, 1970).

Empirically, any line of treatment attempts either to decrease the workload of the left ventricle or to increase the oxygen supply to the ischaemic area. Surgical procedures have been advocated to increase coronary perfusion or to decompress the left ventricle (Kuhn et al., 1968 ; Skinner et al., 1970 ; Bussemaker, Dutka, and Skinner, 1972). However, many of these methods had little success. In the present approach, the oxygen supply to the ischaemic area is directly increased by a simple procedure which seemed effective in improving the manifestation of myocardial ischaemia. In cardiogenic shock, the use of the coronary vein instead of the artery is advantageous. It avoids using cardiac bypass, which could not easily be tolerated in these patients. Secondly, in order to use the artery, accurate outlining of the artery preoperatively is essential in the form of coronary angiography. Lastly, our technique avoids direct manipulation of the artery during the acute attack, which may interfere with any blood flow provided by the collateral circulation.

This study raised two questions. First, what is 
the actual flow pattern after coronary vein perfusion? The other, what will be the long-term changes in the walls of the vein? Further studies, which are in progress at the present time to determine the flow pattern, may answer the first question. As regards long-term changes in the wall of the vein, the available experience with saphenous vein graft showed that subjecting the vein wall to high arterial pressure caused proliferative changes but the vein usually remained patent (Johnson, Auer, and Tector, 1970 ; Vlodaver and Edwards, 1971).

In conclusion, coronary vein perfusion could provide a substantial blood flow in myocardial ischaemia. There is still a need for objective measurements of the value of the procedure. Available information suggests its possible applicability in certain situations in humans.

We gratefully acknowledge the assistance of Professor W. A. Mackey in preparing this manuscript.

\section{REFERENCES}

Adam, M., Mitchel, B. F., Lambert, C. J., and Geisler, G. F. (1972). Long-term results with aorta-to-coronary artery bypass vein grafts. Annals of Thoracic Surgery, 14, 1.

Beck, C. S., Stanton, E., Batiuchok, W., and Leiter, R. (1948). Revascularization of the heart by graft of systemic artery into coronary sinus. Journal of the American Medical Association, 137, 436.

Begg, F. R., Kooros, M. A., Magovern, G. J., Kent, E. M., Brent, L. B., and Cushing, W. B. (1969). The hemodynamics and coronary arteriography patterns during acute myocardial infarction. Journal of Thoracic and Cardiovascular Surgerv, 58, 647.

Bussemaker, J. B., Dutka, M. F., and Skinner, D. B. (1972). Hypothermia and circulatory assistance in dogs with acute myocardial infarction. Journal of Thoracic and Cardiovascular Surgery, 64, 61.
Favaloro, R. G. (1969). Saphenous vein graft in the surgical treatment of coronary artery disease-operative technique. Journal of Thoracic and Cardiovascular Surgery, $\frac{\omega}{\top}$ $58,178$.

- (1970). Surgical Treatment of Coronary Arteriosclerosis. Williams and Wilkins, Baltimore.

Johnson, W. D., Auer, J. E., and Tector, A. J. (1970). Late $\vec{\circ}$ changes in coronary vein grafts (Abstract). American Journal of Cardiology, 26, 640.

- and Lepley, D., Jr. (1970). An aggressive surgical approach to coronary disease. Journal of Thoracic and $\vec{x}$ Cardiovascular Surgery, 59, 128.

Kuhn, L. A. (1970). Shock in myocardial infarction-medical treatment. American Journal of Cardiology, 26, 578.

- Unger, A., Novick, S., Steinhouse, K., Marano, A., $\vec{\omega}$ and Abrams, A. (1968). Effects of combined central $\omega$ aortic counterpulsation and balloon aortic obstruction in myocardial infarction with shock (Abstract). Circu- lation, 38, Suppl. 6, p. 120.

Lassers, B. W., George, M., Anderton, J. L., Higgins, M. R., $\mathbb{D}$ and Philp, T. (1970). Left ventricular failure in acute $\mathbb{D}$ myocardial infarction. American Journal of Cardiology, 25, 511 .

Mitchel, B. F., Adam, M., Lambert, C. J., Sungu, U., and ב Shiekh, S. (1970). Ascending aorta-to-coronary artery saphenous vein bypass grafts. Journal of Thoracic and $\omega$ Cardiovascular Surgery, 60, 457.

Nixon, P. G. F. (1968). Pulmonary oedema with low left ventricular diastolic pressure in acute myocardial infarction. Lancet, 2, 146.

—, Taylor, D. J. E., and Morton, S. D. (1968). Left ventri- ٌٌ cular diastolic pressure in cardiogenic shock treated by dextrose infusion and adrenaline. Lancet, 1, 1230.

Skinner, D. B., Raciti, A., Sabatier, H. S., Newman, M. H., Simon, A. L., and Conti, C. R. (1970). Surgery for acute myocardial infarction: coronary flow and heart work during total circulatory support. Surgery, 68, 128.

Spencer, F. C. (1970). Venous bypass grafts for occlusive disease of the coronary arteries. American Heart Journal, 79, 568.

Vlodaver, Z., and Edwards, J. E. (1971). Pathologic changes in aortic-coronary arterial saphenous vein grafts. Circulation, 44, 719. 